\title{
Avaliação da potência muscular de membros inferiores após realização de protocolo de treinamento neuromuscular e de força muscular
}

\author{
Evaluation of the muscular power of inferior members \\ after performing protocol of neuromuscular training \\ and muscular strength
} \author{
Claudete Rempel ${ }^{4}$ \\ ${ }^{1}$ Fisioterapeuta, Mestre em Fisioterapia - Unit, Professor - Univates - Ulbra. \\ ${ }^{2}$ Fisioterapeutas - Ulbra. \\ ${ }^{3}$ Médico Ortopedista e Traumatologista - Ulbra. \\ ${ }_{4}^{4}$ Bióloga, Doutora em Ecologia - UFRGS, Professora - Univates. \\ Endereço para correspondência \\ Claudete Rempel \\ R. São Pedro, 1190 - Bairro Moinhos \\ 95900-000 - Lajeado - RS [Brasil] \\ crempel@univates.br
}

Fabrício Duarte'; Daisy Valgoi Alca²; Eduarda de Sá Gesser², Fábio Gonçalves Krebs³

\begin{abstract}
Resumo
Neste artigo, analisa-se o ganho de potência muscular de membros inferiores após protocolo de treinamento neuromuscular e de força muscular por meio do teste de Squat Jump, utilizando uma plataforma de força, antes e após treinamentos. A amostra foi composta por 20 voluntários fisicamente ativos, de ambos os sexos, com idade média de 25,3 anos, peso médio de $69 \mathrm{~kg}$, e altura média de $1,71 \mathrm{~m}$. Os indivíduos foram divididos aleatoriamente em três grupos de treinamentos: A) agachamento em superfície estável; B) agachamento em superfície instável; C) neuromuscular utilizando uma prancha de deslizamento. Os participantes dos grupos A e B realizaram teste de uma repetição máxima antes do início dos treinamentos. Encontraram-se diferenças estatisticamente significativas na situação pré e pós-treinamentos, no grupo B e no C. O treinamento em superfície de deslizamento e agachamento em superfície instável foram mais efetivos para o ganho de potência muscular.
\end{abstract}

Descritores: Adaptação neural; Ganho de potência; Treinamento de força.

\begin{abstract}
This work aims to analyze the gain of muscular power of inferior limbs after protocol of neuromuscular training and muscular strength, through Squat Jump test, utilizing a strength platform before and after trainings. The sample was composed of 20 physically active volunteers, from both sexes, with an average age of 25,3 years old, average weight of $69 \mathrm{Kg}$, and average height of $1,71 \mathrm{~m}$. The individuals were divided at random in 3 training groups: A) squat on a stable surface; B) squat on an unstable surface; C) neuromuscular through a slider board. The individuals from groups $\mathrm{A}$ and $\mathrm{B}$ performed the $1 \mathrm{RM}$ test before the beginning of trainings. The volunteers performed training for muscular power gain 3 times a week, during 3 consecutive weeks. Differences were found that were statistically significant among the numbers obtained during pre and post training situations, in group B and in group C. The training on a slider board surface and squat on an unstable surface were more efficient for the gain of muscular power.
\end{abstract}

Key words: Neural adaptation; Power gaining; Strength training. 


\section{Introdução}

Segundo relatos de Hollman et al. ${ }^{1}$, a fisioterapia desportiva tem a função de analisar as influências do movimento, do treinamento e do desporto, assim como o sedentarismo nas pessoas sadias e em doentes de todas as idades. Seu objetivo é aprimorar os resultados da prevenção, da terapia e da reabilitação.

De todas as capacidades biomotoras, a força e a potência são as mais críticas para muitos desportos. Todos os esportes coletivos e os predominantemente de velocidade e potência se apoiam em força e desenvolvimento de potência ${ }^{2}$.

Fleck et al. ${ }^{3}$ relatam que o treinamento de força transformou-se em uma das formas mais conhecidas de exercício, para melhorar tanto o desempenho físico de atletas quanto a prática de indivíduos ativos. Embora a força absoluta seja um componente influente no desempenho físico, a potência - composta de força e velocidade é ainda mais importante para a realização da maioria das atividades. A velocidade é uma qualidade inata que pouco se altera com o treinamento, assim, a potência é aumentada quase que exclusivamente por meio de ganhos de força ${ }^{4}$.

Um treinamento bastante utilizado, com intuito de ganho de força muscular e potência, é o exercício de agachamento, considerado um dos mais completos que podem ser realizados nas academias, envolve elevado número de articulações e músculos, consistindo em excelente meio de fortalecer os músculos do quadríceps, do quadril e muitos outros coadjuvantes que atuam na realização do movimento ${ }^{5}$.

Com relação à carga máxima, há um teste em que se realiza uma tentativa ou uma repetição máxima (1RM) para determinar a força de $100 \%$ dos movimentos primordiais do atleta. Ele possibilita que o treinador conheça a força máxima do indivíduo, decida a porcentagem de carga para o treinamento, de acordo com os objetivos e características do esporte, com as necessidades do atleta e com o tipo de força visada ${ }^{2}$.

Perry $^{6}$ relata que um instrumento muito utilizado para mensurar a potência e a força muscular é a plataforma de força, também usada para medir as forças de reação do solo durante a locomoção humana, saltos e outros movimentos ou posições.

Cronin et al. ${ }^{7}$ afirmam que a medida do desempenho do salto vertical é uma forma bastante comum para a avaliação da força e da potência. Os autores ressaltam as diversas derivações do salto vertical com o objetivo de aferir qualidades neuromusculares e de desempenho distintas de um indivíduo, sendo o teste de Squat Jump muito efetivo para verificar tais capacidades motoras dos membros inferiores.

Segundo Wilmore et al. ${ }^{4}$, com o exercício crônico ocorrem adaptações no sistema neuromuscular cuja extensão depende do tipo de programa de treinamento seguido - o aeróbico, como o jogging e a natação, produzem pouco, ou nenhum, ganho de força e potência muscular, mas ocorrem adaptações neuromusculares importantes com o treinamento de força.

Esta pesquisa é direcionada para os profissionais da área de fisioterapia, especificamente da desportiva, que buscam métodos mais eficazes de treinamentos, objetivando adquirir conhecimentos detalhados sobre o melhor trabalho para promover o ganho de potência muscular em seus pacientes.

A potência muscular é necessária para realizar atividades físicas. Os profissionais da área de fisioterapia precisam desse recurso para promover a completa reabilitação do indivíduo, por esse motivo se justifica este estudo, que objetiva avaliar métodos de treinamentos distintos de potência muscular, comparando ganho de força e potência muscular de membros inferiores por meio de prancha de deslizamento, agachamento em superfície instável e estável.

\section{Materiais e métodos}

Este trabalho constituiu-se de uma pesquisa experimental, sendo selecionados estudantes da Universidade Luterana do Brasil (Ulbra), de ambos os sexos, considerados fisicamente ati- 
vos. A amosta foi constituída de 20 indivíduos divididos aleatoriamente em três grupos de treinamento, com idades entre 22 e 35 anos, peso de $43 \mathrm{~kg}$ a $95 \mathrm{~kg}$, e altura entre 1,47 m e 1,94 m.

O grupo A, composto por oito indivíduos, com idade entre 22 e 29 anos, massa corporal de $48 \mathrm{~kg}$ a $88 \mathrm{~kg}$ e altura entre 1,58 m e 1,84 m.

O grupo B, formado por cinco indivíduos, com idade entre 26 e 35 anos, massa corporal entre $72 \mathrm{Kg}$ e $95 \mathrm{Kg}$, e altura entre 1,73 m e 1,94 m.

Os grupos A e B realizaram treinamento de reforço muscular dos membros inferiores, $\mathrm{O}$ A, por meio de exercício de agachamento, e o B, em conjunto com um disco instável.

Já o grupo C, constituído de sete indivíduos, com idade entre 22 e 25 anos, massa corporal de $43 \mathrm{~kg}$ a $82 \mathrm{~kg}$, e altura entre 1,47 m e 1,80 m realizou treinamento neuromuscular apor intermédio de uma prancha de deslizamento.

Todos os indivíduos receberam e assinaram o Termo de Consentimento Livre e Esclarecido (TCLE), concordando em participar do trabalho. O estudo foi submetido e aprovado pelo Comitê de Ética em Pesquisa (CEP) da Universidade Luterana do Brasil (Ulbra), parecer número 2008588 H, conforme determina a Resolução 196/96 do Conselho Nacional de Saúde (CNS).

Foram excluídos da pesquisa indivíduos sedentários, voluntários que realizaram exercícios de musculação de membros inferiores ou outra prática suplementar durante a fase experimental do trabalho, com discrepâncias em membros inferiores de volume (maior que $5 \mathrm{~cm}$ do lado dominante em relação ao contralateral) e de comprimento (maior que $1,5 \mathrm{~cm}$ ); portadores de deficiência física ou de sequela de doença neurológica, e voluntários que não comparecessem aos treinamentos e testes.

Para os dados do nível de atividade física, utilizou-se o estudo de Matsudo et al..$^{8}$ em que determinaram esse nível de acordo com a frequência e duração das sessões de atividades realizadas, dividindo os sujeitos em muito ativos, ativos, irregularmente ativos e sedentários. Considerando indivíduo ativo aquele que realizasse atividades semanais de, pelo menos, três sessões, com du- ração mínima de 20 minutos, com intensidade vigorosa, ou aquele que somasse 150 minutos semanais de atividade moderada.

Para a avaliação da força e potência muscular de membros inferiores, foi utilizado o teste de salto Squat Jump, tipo de salto vertical que, segundo Komi et al. ${ }^{9}$, Cronin et al. ${ }^{7}$ e Galdi ${ }^{10}$ quando executado da maneira correta, ou seja, o indivíduo com a planta dos pés em contato com a plataforma de força, os membros inferiores semiflexionados a $90^{\circ}$, as mãos na cintura, o tronco ereto, e, sem movimentos prévios, saltando, mantendo os joelhos a $180^{\circ}$ e voltando apoiando os pés sobre a referida plataforma -, é um protocolo bastante fidedigno para avaliar a força e a potência muscular desses membros. Por esse motivo, foi o teste utilizado nesta pesquisa, sendo os dados coletados por meio da plataforma de força da marca Multisprint $1.20^{\circledR}$, antes e após o treinamento de reforço muscular em superfície estável (grupo A), instável (grupo B) e por intermédio da prancha de deslizamento (grupo C).

Os saltos eram invalidados se os participantes não seguissem o citado protocolo do teste de salto Squat Jump,ou seja, executassem o movimento com auxílio de membros superiores; flexão maior ou menor do que $90^{\circ}$ de joelho; flexionassem o joelho após o comando do salto (contramovimento); ou quando por parte do experimentador, ou do voluntário, fosse verificado qualquer desconforto durante a execução do salto.

Após a divisão aleatória dos grupos A, B e C, foi feito o teste de 1RM com cada integrante do grupo A e do B, visto que com o C não foi necessário realizar o teste citado, por não utilizar carga para a execução do treinamento.

Realizaram-se treinos com cada grupo, durante três semanas consecutivas, três sessões por semana, com $100 \%$ de assiduidade, sendo concluídos nove treinamentos, após esse período.

O grupo A teve sessões de exercícios em quatro séries de seis repetições, com $80 \%$ da carga máxima e intervalo de dois a três minutos entre cada série, durante a realização do exercício de meio agachamento (Figura 1). Para angulação 
do agachamento, utilizou-se um anteparo de madeira colocado atrás do sujeito, no momento da execução do exercício, para que não ultrapassasse o limite de amplitude de movimento (ADM) desejado. Esse anteparo também foi utilizado durante a realização do teste de 1RM, antes do início dos treinamentos dos grupos A e B.

Os indivíduos se submetiam a aquecimento de, no mínimo, cinco minutos em bicicleta ergométrica ou corrida em esteira, associado a alongamentos gerais de membros superiores e inferiores, antes de iniciar cada treinamento.

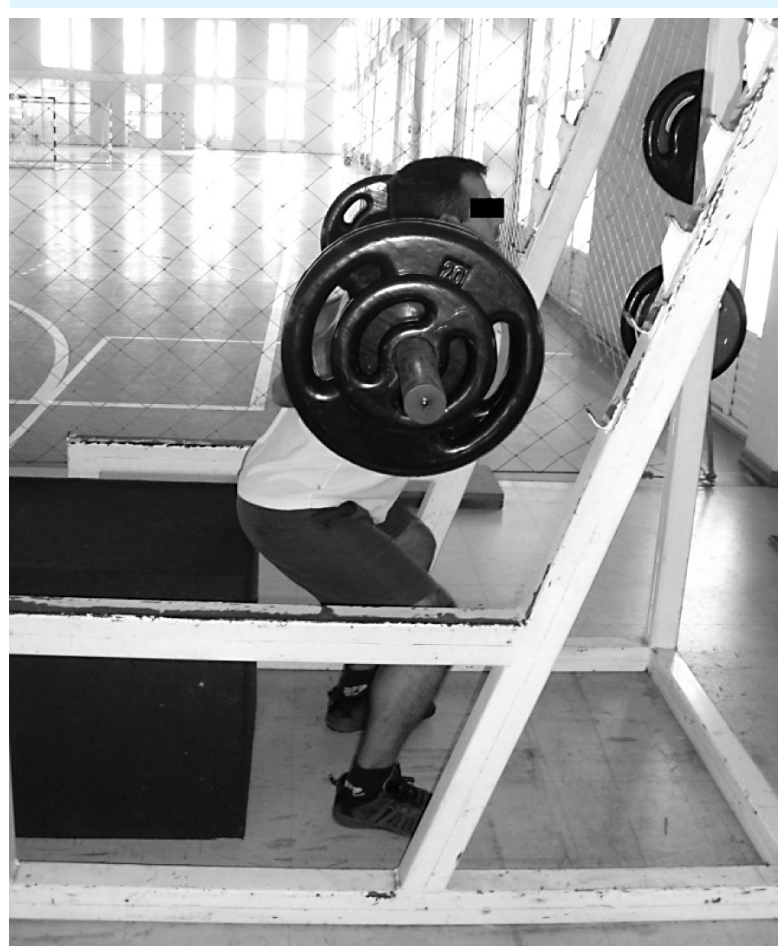

Figura 1: Treinamento de meio agachamento

O grupo B realizou o mesmo procedimento do $\mathrm{A}$, porém apoiando os membros inferiores sob dois discos instáveis, da marca Dyna Disc ${ }^{\circledR}$ (Figura 2).

O grupo $\mathrm{C}$ efetuou treinamento de deslizamento, com oito séries, de 15 segundos, e intervalo de 45 segundos entre cada série, realizando o exercício em uma velocidade constante, e angulação de quadril de aproximadamente $90^{\circ}$, sob uma prancha de deslizamento de $2 \mathrm{~m}$ de largura (Figura 3). Para tal, os voluntários utilizaram uma sapatilha deslizante nos pés.

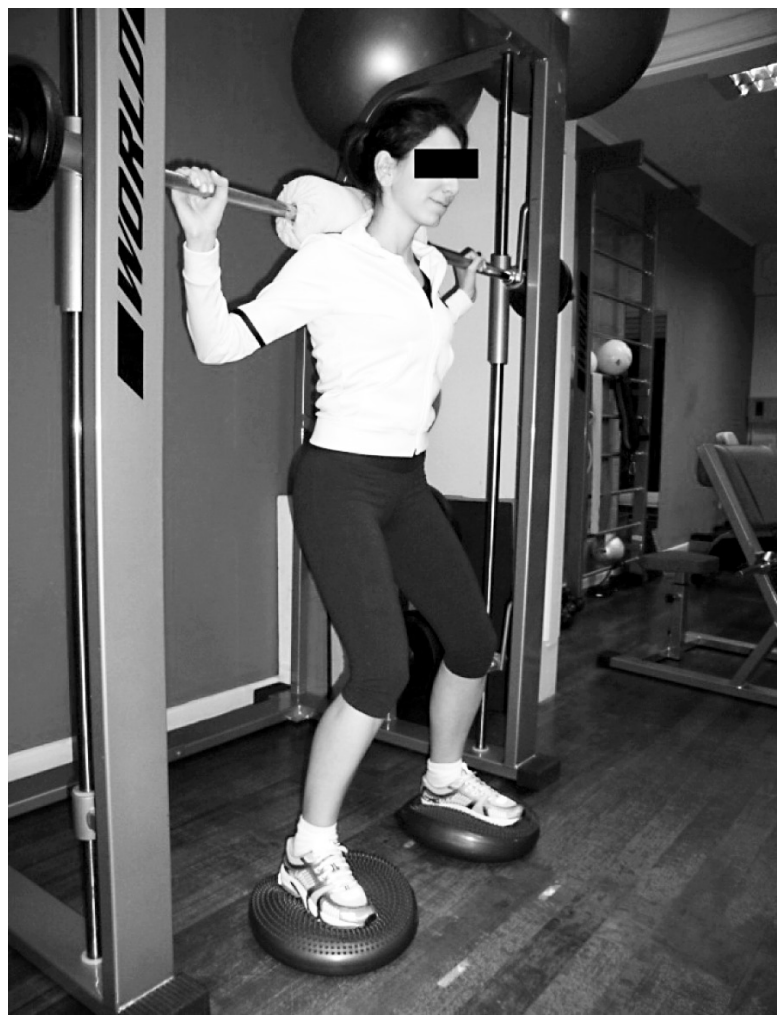

Figura 2: Treinamento de meio agachamento com discos instóveis

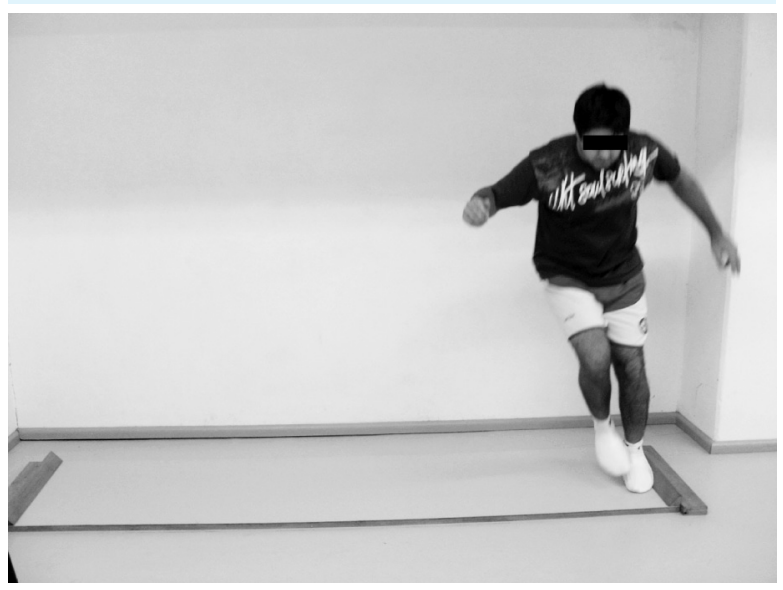

Figura 3: Treinamento sobre prancha de deslizamento

\section{Resultados}

As Tabelas 1, 2 e 3 apresentam os resultados dos testes do pré e pós-treinamento e as variáveis antropométricas - idade, peso e altura dos voluntários dos grupos A, B e C. No grupo 
A, obteve-se a média de resultado do teste de Squat Jump no pré-treinamento de $27,66 \mathrm{~cm} \mathrm{(} \pm$ $5,02)$, e no pós-treinamento, de $29,09 \mathrm{~cm}( \pm 4,36)$. As médias apuradas de idade, peso e altura foram 25,87 anos $( \pm 3,90), 68,12 \mathrm{~kg}( \pm 14,88)$ e $1,70 \mathrm{~m}$ $( \pm 0,08)$, respectivamente.

O grupo B, conforme pode ser observado na Tabela 2, obteve a média de resultado do teste de Squat Jump no pré-treinamento de $34,7 \mathrm{~cm}$ $( \pm 3,68)$, e no pós-treinamento, de $36,48 \mathrm{~cm}( \pm$ $3,16)$. As médias de idade, peso e altura foram 28,2 anos $( \pm 3,83), 82,2 \mathrm{~kg}( \pm 9,80)$, e $1,82 \mathrm{~m} \mathrm{(} \pm$ $0,08)$, respectivamente.

Já a Tabela 3 apresenta os dados do grupo C que obteve a média no teste de Squat Jump no pré-treinamento de $22,25 \mathrm{~cm}( \pm 5,32)$ e no póstreinamento média de $24,57 \mathrm{~cm}( \pm 5,98)$. Neste grupo, identificaram-se as médias de idade, 22,57 anos $( \pm 1,13)$; de peso, $60,57 \mathrm{~kg}( \pm 13,16)$, e de altura, 1,64 $\mathrm{m}( \pm 0,10)$.

Com o objetivo de verificar a existência ou não de diferenças estatisticamente significantes, entre os resultados obtidos na situação pré e na pós-treinamentos, foram aplicados os testes " $\mathrm{t}$ " Student ${ }^{11}$ e de Wilcoxon ${ }^{12}$, considerando-se os três tipos de treinamento: agachamento tradicional, agachamento tradicional sobre uma superfície instável e neuromuscular em uma superfície de deslizamento.

O teste de Wilcoxon foi aplicado quando da comparação entre os resultados obtidos no pré e no pós-treinamento, e no treinamento de agachamento tradicional sobre uma superfície instável, uma vez que a distribuição dos dados relativos à situação pós-treinamento é não normal. O nível de significância foi estabelecido em 0,05 em uma prova bicaudal.

Foram encontradas diferenças estatísticas significativas, por meio de ambos os testes, (considerando um $\alpha=5 \%$ ) entre os valores obtidos nas situações pré e pós-treinamentos, com relação ao treinamento de agachamento tradicional ( $p=0,050)$ em superfície instável $(0,043)$, e ao neuromuscular em superfície de deslizamento $(\mathrm{p}=0,002)$.
Tabela l: Resultado Squat Jump pré e póstreinamento e varióveis antropométricas do grupo A

\begin{tabular}{c|c|c|c|c|c}
\hline Grupo & SJ Pré & SJ Pós & Idade & Peso & Altura \\
\hline A & $(\mathrm{cm})$ & $(\mathrm{cm})$ & (anos) & $(\mathrm{Kg})$ & $(\mathrm{m})$ \\
\hline 1 & 27,1 & 27,1 & 29 & 56 & 1,70 \\
\hline 2 & 27,0 & 28,0 & 22 & 63 & 1,70 \\
\hline 3 & 28,2 & 27,4 & 24 & 48 & 1,58 \\
\hline 4 & 23,5 & 25,2 & 28 & 86 & 1,75 \\
\hline 5 & 30,7 & 33,5 & 23 & 88 & 1,86 \\
\hline 6 & 28,6 & 30,2 & 26 & 76 & 1,78 \\
\hline 7 & 36,7 & 37,2 & 33 & 73 & 1,67 \\
\hline 8 & 19,5 & 24,2 & 22 & 55 & 1,65 \\
\hline Média & 27,66 & 29,09 & 25,87 & 68,12 & 1,70 \\
\hline Dp & $\pm 5,02$ & $\pm 4,36$ & $\pm 3,90$ & $\pm 14,88$ & $\pm 0,08$ \\
\hline
\end{tabular}

Tabela 2: Resultado Squat Jump pré e póstreinamento e varióveis antropométricas do grupo B

\begin{tabular}{c|c|c|c|c|c}
\hline Grupo & SJ Pré & SJ Pós & Idade & Peso & Altura \\
\hline $\mathrm{B}$ & $(\mathrm{cm})$ & $(\mathrm{cm})$ & $($ anos $)$ & $(\mathrm{Kg})$ & $(\mathrm{m})$ \\
\hline 1 & 35,1 & 36,8 & 35 & 90 & 1,86 \\
\hline 2 & 34,3 & 37,6 & 27 & 76 & 1,86 \\
\hline 3 & 37,9 & 38,0 & 26 & 72 & 1,73 \\
\hline 4 & 28,7 & 31,0 & 26 & 95 & 1,94 \\
\hline 5 & 37,5 & 39,0 & 27 & 78 & 1,82 \\
\hline Média & 34,7 & 36,48 & 28,2 & 82,2 & 1,82 \\
\hline Dp & $\pm 3,68$ & $\pm 3,16$ & $\pm 3,83$ & $\pm 9,80$ & $\pm 0,07$ \\
\hline
\end{tabular}

Tabela 3: Resultado Squat Jump pré e póstreinamento e varióveis antropométricas do grupo C

\begin{tabular}{c|c|c|c|c|c}
\hline Grupo & SJ Pré & SJ Pós & Idade & Peso & Altura \\
\hline $\mathrm{C}$ & $(\mathrm{cm})$ & $(\mathrm{cm})$ & $(\mathrm{anos})$ & $(\mathrm{Kg})$ & $(\mathrm{m})$ \\
\hline 1 & 27 & 31,1 & 22 & 64 & 1,69 \\
\hline 2 & 18,7 & 19,8 & 22 & 48 & 1,47 \\
\hline 3 & 18,5 & 20,9 & 22 & 60 & 1,72 \\
\hline 4 & 18,4 & 21,1 & 22 & 43 & 1,65 \\
\hline 5 & 17,2 & 18,9 & 22 & 70 & 1,58 \\
\hline 6 & 25,3 & 26,2 & 25 & 57 & 1,58 \\
\hline 7 & 30,7 & 34 & 23 & 82 & 1,8 \\
\hline Média & 22,25 & 24,57 & 22,57 & 60,57 & 1,64 \\
\hline Dp & $\pm 5,32$ & $\pm 5,98$ & $\pm 1,13$ & $\pm 13,16$ & $\pm 0,10$ \\
\hline
\end{tabular}

A Figura 4 ilustra a diferença entre os três grupos de treinamentos em relação aos resulta- 
dos observados no teste de Squat Jump, no pré e pós-treinamento.

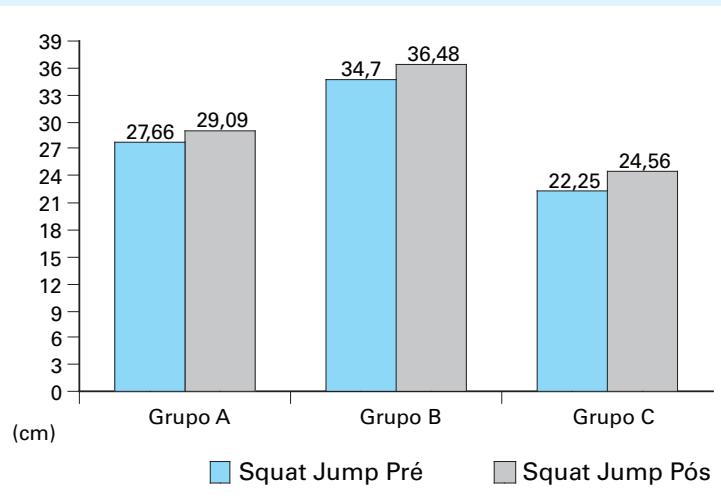

Figura 1: Comparação dos resultados médios, entre os três grupos de treinamento, dos testes de Squat Jump realizados no pré e pós-treinamento

\section{Discussão}

O objetivo neste estudo foi verificar a potência muscular de membros inferiores após protocolo de treinamento neuromuscular e de força muscular. Para tal, foi utilizado o protocolo funcional Squat Jump, que de acordo com Komi et al. ${ }^{9}$, Croning et al. ${ }^{7}$ e Galdi ${ }^{10}$ é bastante usado para a avaliação da força e potência muscular de membros inferiores, pois é de fácil aplicabilidade, não sendo necessário que o indivíduo esteja familiarizado antes de executá-lo.

Oliveira et al. ${ }^{13}$, realizaram o mesmo protocolo, ao analisar a variação da força rápida de membros inferiores de goleiros de futebol júnior, comparando, entre o início e o fim de uma temporada de treinamento, os valores individuais da altura de impulsão vertical máxima. Os autores justificam a melhora no resultado dos testes, pelo fato de o treinamento dos indivíduos consistir em uma sequência de saltos e atividades anaeróbicas aláticas, executadas sempre em velocidades máximas e submáximas.

Em relação à amostragem, os três grupos foram compostos por indivíduos ativos, com média de idade de 25,3 anos $( \pm 3,77)$, estatura mediana de $1,71 \mathrm{~m}( \pm 0,11)$ e massa corporal média de $69 \mathrm{~kg}( \pm 15,13)$. Essa amostra é semelhante à estudada por Furtado et al. ${ }^{14}$ que verificaram o desempenho de atletas de voleibol do sexo feminino, em saltos verticais, em voluntárias com médias de idade 21,2 anos $( \pm 2,54)$, estatura mediana de $1,68 \mathrm{~m}( \pm 0,06)$ e massa corporal média de $66,2 \mathrm{~kg}( \pm 6,73)$.

Baseando-se em Jones et al. ${ }^{15}$ foram identificadas três fases que constituem o processo de adaptação ao treinamento de força. A primeira fase é resultante de um processo de aprendizado, em que se observa pouco aumento no tamanho ou força dos músculos. Na fase dois, ocorre um aumento na força das fibras individuais, gerando um ganho rápido de força muscular. Verificase na fase três, após 12 semanas de treinamento, um aumento lento, porém constante, da força e do tamanho dos músculos exercitados.

O treinamento utilizado para o grupo A nesta pesquisa, corrobora os estudos de Mollet $^{16}$ em que relata que o trabalho para ganho de potência muscular deve ser realizado, inicialmente determinando a RM do indivíduo, e depois, melhorando a velocidade de execução que, quando satisfatória, deve-se aumentar a carga até o indivíduo conseguir completar apenas seis repetições. O intervalo de descanso deve ser de 2 a 3 minutos, quando se exercita com uma carga abaixo de $85 \%$, e de 3 a 5 minutos, quando se excede essa quantidade. Todos os exercícios devem ser realizados com a maior velocidade possível.

Segundo Mollet $^{17}$, para a realização de um treinamento de potência muscular são necessárias cargas entre $80 \%$ a $85 \%$ da capacidade máxima do indivíduo. Os exercícios devem ser executados com repetições baixas, utilizando seis repetições, quando a carga estiver mais elevada, e no máximo 12, com cargas menos elevadas.

$\mathrm{O}$ treinamento do grupo $\mathrm{C}$ aqui proposto ratifica os trabalhos de Boyle ${ }^{18}$ que relata que ocorrem ganhos de força e potência muscular de membros inferiores, quando o exercício de deslizamento é praticado em grande velocidade e de maneira constante, executado sobre pranchas, durante 15 a 20 segundos, seguido de um 
intervalo de descanso de 45 a 60 segundos, e efetuadas de 8 a 10 séries de repetições. O deslizamento deve ser realizado com o joelho em flexão de $90^{\circ}$ a $120^{\circ}$, para haver um maior recrutamento das musculaturas e execução rápida dos movimentos, gerando maior velocidade e potência, durante a prática do exercício.

\section{Resultados}

Em relação aos resultados, foram encontradas diferenças estatisticamente significativas comparando-se os testes realizados no pré-treinamento com os efetuados no pós-treinamento do grupo B (meio agachamento em superfície instável) e grupo C (prancha de deslizamento). Tais resultados podem ser facilmente explicados quando recorremos a Bompa ${ }^{2}$ que explica que o Sistema Nervoso Central (SNC) tem duas ações básicas, a excitação e a inibição, sendo o treinamento muscular responsável por grande variação entre esses dois processos. Durante uma atividade física ocorre uma estimulação do $\mathrm{SNC}$, que envia um número de impulsos nervosos para a musculatura, ordenando que ela se contraia e realize o trabalho, sendo então a velocidade, a potência e frequência do impulso nervoso dependentes do estado do SNC. A força de contração e o número de unidades motoras diretamente recrutadas têm relação direta com a ativação elétrica do SNC; portanto, a mudança persistente na estrutura da função do sistema nervoso e muscular ocorre em resposta a aumentos da carga de treinamento.

Um dado relevante foi que o grupo $C$ (prancha de deslizamento), obteve o maior resultado estatístico verificado em comparação aos testes realizados no pré e pós-treinamento $(p<0,002)$. Tais ganhos podem ser explicados quando consideramos os estudos de Canavan ${ }^{19}$, em que cita que as adaptações específicas também estão associadas às velocidades específicas do movimento, ou seja, para aumentar a força, a velocidade do movimento do exercício também deve ser aumentada. Treinamentos que utilizam exercícios explosivos rápidos, em que se valoriza a velocidade do movimento, resultam em uma melhora substancial na produção de energia máxima, isso ocorre em virtude da velocidade, na qual a força pode ser desenvolvida.

Segundo Kanehisa et al. ${ }^{20}$, o treinamento em uma velocidade rápida resulta em ganhos de força e potência ligeiramente maiores do que o efetuado em uma velocidade lenta, e gera informações neurais mais velozes.

O grupo $\mathrm{B}$ (meio agachamento em superfície instável), realizou treinamento de força acompanhado de estímulo neuromuscular, obtendo melhora comparando os testes realizados no pré com os do pós-treinamento, tendo um resultado estatisticamente significativo $(\mathrm{p}<0,043)$. Strojnik et al. ${ }^{21}$ relataram que o treinamento funcional sobre superfície instável é um importante parâmetro para a reabilitação e condicionamento neuromuscular, e proporciona a melhora da sua coordenação e do padrão de recrutamento. Esse tipo de treinamento auxilia na maior ativação do sistema proprioceptivo em uma atividade principalmente estática com ação das fibras aferentes, porém muitos estudos mostram seu potencial na execução dinâmica ${ }^{22,23}$.

De acordo com Maior et al. ${ }^{24}$, quando ocorre a adaptação neural os impulsos excitatórios excedem os inibitórios da fibra muscular, dando início a contração do músculo. Este procedimento facilita, de forma benéfica, o recrutamento de unidades motoras ativadas sincronicamente, o aumento da movimentação do motor central e a maior excitabilidade dos motoneurônios. O treinamento com instabilidade gera, por meio da adaptação neural, habilidade para mais coordenação do movimento e estabilização, aumentando a produção de força.

O grupo A (meio agachamento em superfície estável) não obteve melhora estatisticamente significativa $(p=0,050)$. Este resultado contradiz os encontrados por Lamas et al. ${ }^{25}$ que realizaram treinamento com indivíduos saudáveis, fisicamente ativos, com pelo menos seis meses de interrupção no treinamento de força para membros inferiores. Os sujeitos dos grupos de treina- 
mento de força (TF) e potência (TP) foram submetidos a oito semanas de exercícios, com três sessões semanais. Os grupos TF e TP aumentaram a força e potência de membros inferiores estatisticamente de forma similar. Portanto, o treinamento realizado neste estudo, com o grupo A, que utilizou o exercício de meio agachamento durante três semanas, três sessões por semana, pode não ter tido resultado estatisticamente significativo por não haver realizado o tempo de treinamento e número de sessões necessário para o ganho de força e potência muscular.

Segundo Bompa ${ }^{2}$ e Komi ${ }^{26}$, o treinamento muscular realizado para ganho de potência, se realiza por intermédio de uma contração do tipo alongamento-encurtamento, que em conjunto com um impulso nervoso, durante a fase concêntrica, produz uma impulsão potente. A adaptação do sistema nervoso aos estímulos de treinamento desempenha um papel importante na atividade motora, já que ele reage sensitivamente para adaptar estímulos contráteis rápidos ou lentos. Os exercícios de potência resultam em uma rápida mobilização de grandes atividades de inervação, no recrutamento da maioria das unidades motoras e suas fibras musculares correspondentes, e num aumento no índice de descarga de neurônios motores. Esse aumento na inervação produz melhoras consideráveis no desenvolvimento de potência ${ }^{27,28,29,30}$.

De acordo com Canavan ${ }^{19}$ e Bompa ${ }^{2}$, a fisiologia neuromuscular é responsável pelos estímulos neurais, que estabelecem padrões para o movimento cinético, para as secções enzimáticas dos músculos e para a composição do tipo de fibra muscular. Os exercícios realizados com cargas elevadas são específicos e resultam em um aumento da força máxima. Quando, ao contrário, realiza-se um programa de treinamento para ganho de força explosiva, melhoram-se os resultados da produção desta, porém com pouco aumento da força máxima. A relação força muscular e velocidade é vista como uma relação inversa: à medida que o percentual de carga aumenta a força também aumenta; a velocidade, porém, diminui. Uma vez que essa relação é aplicada no músculo humano, os resultados diferem significativamente, o que pode ser explicado pelo fato de os proprioceptores serem sensíveis à força e por prevenirem lesões causadas pelo nível de força.

\section{Conclusão}

Por meio deste estudo, pode-se concluir que o treinamento de potência muscular em superfície instável e com pranchas de deslizamento obtiveram melhora significativa no ganho de força e potência de membros inferiores. Além da utilização de treinamentos tradicionais, verificou-se que o realizado com instabilidade possibilita, por intermédio de adaptações neurais, ganho de coordenação e estabilização, aumentando a produção de força e potência muscular. O executado com pranchas de deslizamento promovem um aumento da força muscular pela velocidade elevada de execução de movimento, já o treinamento explosivo rápido, resulta em uma melhora substancial de informações neurais e de produção de potência muscular.

\section{Referências}

1. Hollman W, Hettinger T. Medicina do esporte. Edição Revisada. São Paulo: Manole; 1989.

2. Bompa TO. Treinamento de potência para o esporte: desenvolvimento máximo da potência. São Paulo: Phorte; 2004.

3. Fleck SJ, Kraemer WJ. Fundamentos de treinamento de força muscular. $2^{\text {a }}$ ed. Porto Alegre: Artes Médicas; 1999.

4. Wilmore JH, Costill DL. Fisiologia do esporte e do exercício. $2^{a}$ ed. São Paulo: Manole; 2001.

5. Oliveira E, Gentil P. Musculação: Posto de Vista agachamento. Saúde em Movimento. 2002; [s.v.]:Não paginado. [acesso em: 2009 jun. 14]. Disponível em: http://www.saudeemmovimento.com.br/conteudos/ conteudo_exibe1.asp?cod_noticia=843.

6. Perry J. Análise da marcha humana. São Paulo: Manole; 2005. 
7. Cronin JB, Hing, RD, Mcnair, PJ. Reability and validity of a linear position transducer for measuring jump performance. J Strength Cond Res. 2004;[s.v.]:590-603.

8. Matsudo SM, Matsudo VR, Araújo T, Andrade D, Andrade E, Oliveira L, et al. Nível de atividade física da população de São Paulo: análise de acordo com o gênero, idade, nível socioeconômico, distribuição geográfica e de conhecimento. Revista Brasileira Cineantopometria e Movimento de Brasília. 2002;10(4):41-50.

9. Komi PV, Bosco C. Utilization of stored elastic energy in leg extensor muscles by men and women. Medicine Science and Sports Exercise. 1978;10(4):261-5.

10. Galdi EHG. Pesquisas com salto vertical: uma revisão. Revista Treinamento Desportivo. 2000;1(1):27.

11. Graner EA. Estatística. São Paulo: Melhoramentos; 1966.

12. Siegel S. Estatística não-paramétrica, para ciências do comportamento. Trad. Alfredo Alves de Farias. São Paulo: McGraw-Hill do Brasil; 1975.

13. Oliveira AL, Siqueira OD, Padilha JR. Acompanhamento da força rápida em goleiros de futebol júnior no primeiro semestre de competição. Revista Digital. 2004;10(75): http://www.efdeportes.com/

14. Furtado GS, Melo RRO de, Garcia MAC. Desempenho de atletas de voleibol do sexo feminino em saltos verticais. Arquivos em Movimento. Revista Eletrônica da Escola de Educação Física e Desporto. 2006; 2(2):[s.p.].

15. Jones DA, Rutheford OM, Parker DF. Physicological changes in skeletal muscle as a result of strength training. QJ Exp. Physiological. 1989; [s.v.]:233-56.

16. Mollet R. Power Training. Brussels: Cross Promenade; 1963.

17. Mollet R. Treinamento de Força. São Paulo: Fórum; 1972.

18. Boyle M. Sliding Through. Training \& Conditioning. 2001; 11(7):[s.p.]. http://www.momentummedia.com/ articles/tc/tc1107/sliding.htm

19. Canavan PK. Reabilitação em Medicina Esportiva. São Paulo: Manole; 2001.

20. Kanehisa H, Miyashita M. Effect of isometric and isokinetic muscle training on static strength and dynamic Power. Eur J Appl Pshysiol. 1983; 50:365-371.
21. Strojnik V, Vengust R, Pavlovcic V. The effect of proprioceptive training on neuromuscular function in patients with patellar pain. Cell Mol Biol Lett. 2001;7(1):170-1.

22. Heitkamp HC, Horstamann T, Mayer F, Weller J, Dickruth HH. Gain in strength and muscular balance after balance training. Int J Sports Med. 2001;22(4):285-90.

23. Soderman K, Werner S, Pietila T, Engstrom B, Alfredison $\mathrm{H}$. Balance board training: prevention of traumatic injuries of the lower extremities in female soccer players? A prospective randomized intervention study. Knee Surg Sports Traumatol Arthrosc. 2000;8(6):356-63.

24. Maior AS, Moraes ER, Santos TM, Simão R. Análise da Força Muscular em indivíduos treinados na plataforma de instabilidade. Revista Brasileira de Ciência e Movimento. 2006;14(2):41-8.

25. Lamas L, Ugrinowitsch C, Campos GER, Aoki MS, Fonseca R, Regazzini M, et al. Treinamento de força máxima $x$ treinamento de potência: alterações no desempenho e adaptações morfológicas. Revista Brasileira de Educação Física e Esporte. 2007;21(4):331-40.

26. Komi PV. Força e Potência no Esporte. $2^{\circledR}$ ed. Porto Alegre: Artmed; 2006.

27. Clutch D, Wilton M, Mcgown C, Bryce T. The effect of depth jumps and weight training on leg strength and vertical jump. Research Quarterly for Exercise \& Sport. 1983;54(1):5-10.

28. Gollhofer A, Komi PV, Miyashita M, Aura O. Fatigue during stretch-shortening cycle exercises: changes in mechanical performance of human skeletal muscle. International Journal of Sports Medicine. 1987;8(2):71-8.

29. Gollhofer A, Fujitsuka N, Miyashita M. Fatigue during stretch-shortening cycle exercises II. Changes in neuromuscular activation patterns of human skeletal muscle. International Journal of Sports Medicine. 1987;8:38-47.

30. Schmidtbleicher D, Gollhofer A. Neuromuscular examination of the determination of individual training loads for jump training. Leistungssport. 1982;12(4):298-307. 
